\title{
La Décentralisation Face à l'Ordre Ancien
}

Soli Koné

\section{CpenEdition}

\section{Journals}

Édition électronique

URL : http://journals.openedition.org/apad/586

DOI : 10.4000/apad.586

ISSN : 1950-6929

\section{Éditeur}

LIT Verlag

Édition imprimée

Date de publication : 1 décembre 1997

\section{Référence électronique}

Soli Koné, «La Décentralisation Face à I'Ordre Ancien », Bulletin de I'APAD [En ligne], 14 | 1997, mis en ligne le 29 janvier 2007, consulté le 07 septembre 2020. URL : http://journals.openedition.org/apad/ 586 ; DOI : https://doi.org/10.4000/apad.586

Ce document a été généré automatiquement le 7 septembre 2020.

Bulletin de I'APAD 


\title{
La Décentralisation Face à l'Ordre Ancien
}

\author{
Soli Koné
}

1 Le processus de décentralisation au Mali, s'est véritablement concrétisé sur le terrain dès les premières années de la 3ème République. Cette mise en œuvre effective a mis fin aux velléités et hésitations des 1ère et 2ème Républiques.

2 La démocratisation de la vie politique au Mali à partir de 1991 et la volonté des responsables politiques $d u$ jour sont des facteurs militant en faveur des réformes socio-politiques permettant aux populations à la base de participer à l'exercice du pouvoir et de gérer leur propre développement. Depuis quelques années déjà, le Mali vit le passage d'un Etat centralisé où toutes les décisions concernant la vie des populations relevaient des prérogatives du sommet, à l'exercice démocratique du pouvoir à la base, pour que les collectivités elles-mêmes décident de leurs affaires, en vue d'un développement harmonieux. La Mission de décentralisation, l'organisme chargé de la mise en œuvre de la réforme a mis en place des équipes pour sensibiliser les populations, leur expliquer dans les détails ce qu'est la décentralisation.

Bien que n'étant pas initiatrices, les populations ont fait preuve de beaucoup d'enthousiasme lors des passages de ces équipes dans un premier temps. La seconde étape consistera en la formation des communes à partir d'un certain nombre de critères. On procédera ainsi au découpage du territoire en communes urbaines et rurales. Cependant, les différentes interprétations des messages des équipes de sensibilisation, le découpage, le choix des chefs-lieux de communes ont créé des situations conflictuelles au sein des populations rurales. En effet l'exercice du pouvoir à la base pose un certain nombre de problèmes. Il existe une survivance tenace des structures traditionnelles $\mathrm{du}$ pouvoir et d'une organisation sociale fortement hiérarchisée. La gouvernance libre, sinon autonome des collectivités décentralisées, fait appel au choix démocratique des responsables. Cela ne semble pas bien compris ou accepté de tous. Pour les uns, il s'agit d'un retour à l'organisation socio-politique traditionnelle fortement hiérarchisée fondée sur le principe d'antériorité et/ou sur les conquêtes, pour d'autres c'est l'occasion d'un nivellement et de promotion pour tous 
les acteurs sociaux. Une divergence de vue se cristallise vite en opposition entre les tenants du pouvoir traditionnel, partisans de l'ordre ancien et les autres lignages et groupes sociaux de la zone, partisans de la réforme, à savoir la décentralisation. Cette différence de perception va exercer une influence préjudiciable au bon déroulement des opérations de découpage territorial. Forts de leurs prérogatives dans les domaines politique et foncier, les détenteurs des chefferies traditionnelles ont souvent eu une attitude négative dans la formation de communes apparemment viables. Nous retenons donc comme hypothèse de travail que :

Les difficultés rencontrées dans les opérations de découpage ont été très souvent générées par la résistance de l'ordre ancien par rapport à la réforme que constitue la décentralisation. L'analyse de la phase de découpage et de toutes ses péripéties permettra certainement de faire ressortir les différents aspects du rapport conflictuel entre ordre ancien et décentralisation. Pour se faire, nous nous sommes intéressés au cas de l'arrondissement de Fana et particulièrement à la commune de Tinkolé.

Histoire d'une décentralisation Cadre socio-politique

5 La constitution de 1960 voulait opérer une décentralisation à tous les échelons en créant des assemblées délibératives auprès de chacun d'eux. C'est ainsi que des assemblées régionales élues au suffrage universel étaient prévues, ces assemblées n'ayant pas été établies, des commissions spéciales comprenant les commandants de cercle et les chefs de services régionaux se réunissaient annuellement pour la préparation des budgets régionaux. En 1977 l'ordonnance n 77-44/CMLN du 22 juillet, érigea Bamako en un district subdivisé en six communes dotées du statut de collectivités territoriales décentralisées. Aussi la loi n88-65/AN-RM du 16 mars 1988 portant ouverture du budget de l'Etat d'un compte d'affectation spéciale dénommé "Fonds de développement régional et local" est intervenue pour l'équilibre des régionaux et locaux. Il faut admettre que les différents textes pris en faveur de la décentralisation à cette époque étaient limités dans leur application.

Depuis les événements de mars 1991, le Mali s'est engagé dans la voie de la démocratie caractérisée par un multipartisme intégral. Très tôt, les réformes dans le domaine socio-politique se sont révélées indispensables pour la consolidation de cette démocratie. En effet, l'une des recommandations de la Conférence nationale était d'instaurer un mode de gouvernance qui soit conforme au régime démocratique et favorable à un développement harmonieux à la base. Il faut signaler que l'instauration du processus démocratique comme celle de la décentralisation correspondait au point de vue des institutions internationales à savoir la Banque Mondiale le FMI et les différents bailleurs de fonds, partenaires du Mali pour son développement. Pour de nombreux auteurs, la décentralisation contribue plus au développement économique général que les régimes fortement centralisés (Hyden 1984. Wunsch \& Olowu 1992) cités par Richard Vengroff dans La décentralisation en Afrique: Revue de la littérature.

Cadre Technique et Juridique

7 Les premières communes du Mali appartiennent à la période coloniale. En effet les communes de Kayes et de Bamako du Soudan Français (ancienne appellation du Mali) ont été créées dès 1918 sous le titre de commune mixte du premier degré. A l'indépendance, la République du Mali comptait cinq communes de plein exercice. La loi 66-9/PM-RM du 28 mars 1966, portant code municipal au Mali a donné le statut de commune de plein exercice à toutes les communes. Le Mali jusqu'à une date récente comportait 19 collectivités décentralisées uniquement dans les centres urbains. C'est 
surtout à partir de 1993 que la décentralisation va s'étendre à tout le pays avec la constitution des communes rurales. La volonté de créer des collectivités décentralisées à la base se concrétisera dans un premier temps par la mise en place d'instruments techniques et d'un cadre juridique.

Les Instruments Techniques

8 La mise en œuvre du projet de réforme ne deviendra une réalité qu'avec la création d'un instrument appelé Mission de décentralisation par décret n93-001/PM-RM du 6 janvier 1993 pour un an rattachée au ministère de l'Administration Territoriale. Un an après, le mandat de cette institution fut prolongé de trois ans par décret $\mathrm{n}^{\circ}$ 94-051/ PM-RM du 26 janvier 1994 et elle fut rattachée à la Primature. Elle se dota de quatre structures :

- Une cellule chargée de la création des collectivités;

- Une cellule chargée du développement des collectivités ;

- Une cellule chargée du découpage territorial ;

- Une cellule chargée de la formation des élus.

La Mission de décentralisation sous la responsabilité de son chef a bénéficié du concours de groupes informels d'hommes et de femmes choisis pour leur valeur intrinsèque, dans l'exécution de certaines de ses tâches. Il s'agit des GREM (Groupe régional d'étude et mobilisation au niveau régional) GLEM (Groupe local d'étude et de mobilisation au niveau .du .cercle), GAEM (Groupe d'arrondissement d'étude .et de mobilisation au niveau de l'arrondissement). Il y eut aussi des équipes de découpage, d'arbitrage et de réconciliation. Elle n'a entamé le processus que dans un cadre tracé par des textes législatifs et réglementaires.

Les Instruments Juridiques

10 Lorsqu'on se réfère à la constitution du Mali il est dit dans son article 70 que la loi détermine les principes fondamentaux relatifs à la libre administration des collectivités locales, à leurs compétences, à leurs ressources, ainsi qu'à l'organisation administrative $\mathrm{du}$ territoire. Les dispositions dans ce sens se sont traduites par un ensemble de textes législatifs dont l'énumération serait fastidieuse ici. Nous nous contenterons d'en citer quelques-unes à titre d'exemple.

11 La première en la matière est la loi n 93-008/PM-RM du 11 février 1993 déterminant les conditions de la libre administration des collectivités territoriales. Elle reconnaît comme collectivités: les régions, le district de Bamako, les cercles, les communes urbaines et rurales. Elle institue trois niveaux d'autorité décentralisée : les régions sont subdivisées en cercles, et ces derniers en communes urbaines et rurales. Les communes se composent de villages, quartiers et fractions.

12 La loi $\mathrm{n}^{\circ}$ 95-022/PM-RM du 12 avril 1995 portant code des collectivités territoriales dit dans son article 14 "le conseil communal règle par ses délibérations, les affaires de la commune, notamment celles relatives aux programmes de développement économique, social et culturel. Elle confère de larges compétences aux autorités élues des collectivités territoriales décentralisées en matière de gestion des ressources naturelles et foncières. Elle détermine essentiellement les matières à décentraliser en terme de compétences et de ressources au niveau de la commune, du cercle et de la région.

13 La loi du 16 octobre 1996 portant principes de constitution et de gestion du domaine des collectivités territoriales fait la différence entre domaine public et domaine privé 
dans une collectivité territoriale : (article 1) "le domaine des collectivités territoriales se compose de l'ensemble des biens meubles et immeubles acquis à titre onéreux ou gratuit par lesdites collectivités ou attribués à celles-ci par la loi". La loi n 96-059 du 4 novembre 1996 portant création des communes achève la réorganisation territoriale de la République du Mali. Ce sont quelques-unes des lois à l'intérieur desquelles les activités relatives à la décentralisation vont être menées.

Les étapes de la mise en œuvre de la décentralisation La phase de sensibilisation Les GREM,GLEM et les GAEM, bien qu'étant des groupes informels ont constitué une ossature essentielle dans cette phase. C'est sur ces groupes que la Mission de décentralisation s'est appuyée pour exécuter toutes les tâches qu'elle a déjà accomplies sur le terrain. Les travaux ont consisté à :

- expliquer ce qu'est la décentralisation aux populations, les sensibiliser pour obtenir leur adhésion,

- expliquer les motivations profondes de cette réforme,

- expliquer les relations entre démocratie, décentralisation et développement,

- expliquer les bases sur lesquelles les villages peuvent s'organiser pour former des groupes viables, qui deviendront des communes.

Cependant ces groupes n'ont pu jouer ce rôle que grâce à une formation assurée par la Mission de décentralisation. En effet, il y a eu une formation en cascade : les membres des GREM ont été formés par le personnel de la Mission, ceux-ci à leur tour les membres des GLEM qui ont par la suite formé ceux des GAEM. Nantis de ces informations, ils ont pu porter les messages auprès des populations. Il faut signaler que le personnel de commandement n'a pas été associé dans la plupart des cas à cette phase. Les raisons de cette exclusion n'ont jamais été explicitées. On peut imaginer tout simplement que l'administration centrale n'était pas bien placée pour parler de décentralisation territoriale. Aussi, les différents groupes n'avaient d'autres prérogatives que de convaincre les populations et avoir leur adhésion à la réforme. Il n'était permis à aucun des groupes d'exercer un quelconque pouvoir, du moins théoriquement. Il serait intéressant de voir si les choses se sont passées comme ça ou pas, car les hommes et les femmes formant ces groupes étaient pour certains, responsables de partis politiques. A la fin de la phase de sensibilisation, les populations devaient procéder durant deux à trois mois, à des concertations pour la constitution des groupes de villages, les futures communes.

La phase de découpage

16 Si la première phase a suscité beaucoup d'enthousiasme auprès des populations, les opérations de découpage n'ont pas été sans difficultés à maints endroits du pays pour des raisons diverses. Devant permettre la formation des communes, des critères définis par la Mission constituaient le cadre de leur réalisation. En effet, les critères au nombre de cinq étaient les suivants :

- critères socio-culturels (les solidarités communautaires, les affinités sociales et culturelles etc) ; ce critère est très important car il constitue une base essentielle de la formation des communes ;

- critères démographiques (une commune devait compter de 5.000 à 10.000 habitants) ;

- critères de distance et d'accessibilité se rapportant au chef-lieu de commune ;

- critères de viabilité économique (les ressources naturelles agricoles, présence d'une foire, de projet de développement ou d'ONG); 
- critères géographiques et spatiaux : une commune doit constituer un territoire cohérent et unitaire.

17 Les critères fixés en dehors des populations qui ne correspondaient pas toujours au point de vue de ces dernières, ont provoqué des blocages dans le processus de découpage à travers le territoire. Le souci de la Mission de constituer des groupes viables, exprimé par les critères était-il mal perçu ? Les populations voyaient-elles là un schéma qu'on voulait leur imposer? Ces questions se rapportent à des aspects certainement importants à étudier, toujours est-il que les réactions des populations ont abouti à la formation de micro-communes, de deux à trois villages par endroit, avec un nombre d'habitants bien en deçà du seuil de découpage. Celui-ci a été le révélateur d'aspects non moins importants, assez complexes et tout à fait conflictuels. Nous estimons qu'une étude de cas permettrait de mieux analyser ces aspects. Nous nous proposons d'étudier les problèmes liés au découpage dans l'arrondissement Fana, particulièrement dans la commune de Tinkolé, appelée commune Bêkan.

Découpage et situation conflictuelle dans la commune de TinkoléLa Commune de Tinkolé

18 Tinkolé, village situé à $19 \mathrm{~km}$ de Fana est le chef-lieu d'une des sept communes que compte l'arrondissement. Cette commune se compose de douze villages. Comme d'autres communes, celle de Tinkolé connaît des problèmes dans sa composition elle-même. En effet certains villages sont demeurés réfractaires à la constitution de la commune autour de Tinkolé comme chef-lieu. Les différentes visites des équipes d'arbitrage n'y ont rien changé. Pour mieux comprendre les causes profondes d'un tel refus nous sommes allés sur le terrain auprès des différents acteurs. Les arguments de part et d'autre sont d'ordre historique et socio-politique.

Les aspects historique et socio-politique

19 L'existence des textes législatifs consacrant l'émergence de 701 communes (communes urbaines et rurales confondues) n'a pas mis fin aux attitudes réfractaires, dans certaines d'entre elles. La persistance de ces problèmes, pourrait trouver son explication dans le passé de ces contrées. En effet, trois villages sur une douzaine que compte la commune ne se considèrent pas comme membres de cette entité, malgré toutes les tentatives de réconciliation, parce que Tinkolé en est le chef-lieu. Il s'agit de Fougani, Kalaban et Sanankoro.

20 Historiquement, la commune de Tinkolé est une portion de la partie sud-ouest d'une zone anciennement appelée Guényeka ou Gwenka (Monteil 1977). Le Guényéka était une province du royaume de Segou, limitée au nord par Dnandougou, au nord-est par le Segoudougou, au sud par le Baniko, à l'ouest par le Méguétan. Les trois villages cités ci-dessus se considèrent comme faisant partie des plus anciens, en tout cas établis là, avant Tinkolé. Donc le choix du chef-lieu consécutif au découpage, ne devait pas tomber sur ce dernier. En effet lorsqu'on se réfère à l'histoire du peuplement de la zone, Fougani est non seulement plus ancien, mais aurait offert son hospitalité à ceux de Tinkolé sur ses terres. Les fondateurs de Tinkolé reconnaissent qu'ils sont les hôtes des Coulibaly de Fougani et que toutes leurs terres, leur ont été léguées par ces derniers. Dès lors, on mesure toute la frustration ressentie à Fougani et Kalaban, deux villages créés par le même lignage. Quant aux Coulibaly de Sanankoro, ils avancent les même raisons pour justifier leur attitude. Le choix de Tinkolé leur apparaît comme une espèce d'assujettissement inacceptable compte-tenu de l'histoire de ces communautés. Est-ce là une mauvaise interprétation des messages distillés lors du découpage ou une 
perception confuse de la décentralisation? Pour les Coulibaly de Fougani, si les populations à la base doivent désormais prendre les décisions concernant leur propre développement, le fait d'être parmi les premiers occupants, aurait dû servir à quelque chose dans le choix du chef-lieu. Il y a là résurgence d'un principe essentiel de l'ordre ancien, propre à l'organisation des sociétés traditionnelles face au processus de.la décentralisation.

21 Le principe d'antériorité demeure chez les Bamanan de cette zone, un élément important de l'organisation sociale, son non respect constituant une déviation tout à fait préjudiciable à toute vie de société. Le chef-lieu leur apparaît comme le pôle politique le plus important, aussi bien dans le cadre de l'organisation sociale traditionnelle que dans celui de l'administration moderne, car c'est de là qu'émanent les décisions. Recevoir les décisions de Tinkolé (un village qu'ils ont installé) leur est si insupportable qu'ils ne perçoivent pas leur caractère collégial au niveau communal.

Au plan socio-politique les Coulibaly de Fougani et de Kalaban se disent descendants de grands guerriers ayant joué les premiers rôles dans la défense de la zone contre des envahisseurs venant du Dièmadougou ou du Méguetan. En collaboration avec Soukoubili, Fougani et kalaban ont su protéger de vastes étendues de terres sur lesquelles nombre de villages de la commune sont installés actuellement. Ils estiment avoir détenu un pouvoir politique, même s'ils n'ont pas exercé une domination aussi marquée sur les autres, toute chose que les Fama de Segou (maîtres incontestés du Guényéka) n'auraient pu tolérer. Ils pensent non seulement que Fougani aurait dû être choisi comme chef-lieu, mais qu'eux- mêmes auraient dû aussi bénéficier de privilèges lors de la mise en place des responsables de la commune. Les fondateurs de fougani, nantis de ce passé socio-politique quelque peu glorieux, se sentent plus aptes à l'exercice du pouvoir que d'autres qu'ils ont protégés ou reçus en hospitalité. Leur attachement à cet ordre ancien est tel, qu'ils ont refusé certaines propositions de l'équipe d'arbitrage. En effet, à défaut de s'associer à la commune de Tinkolé, elle leur a proposé de s'ajouter à celle de Marka-gounko. Leur refus fut encore plus marqué car selon eux, les fondateurs de ce village sont les descendants de leurs dépendants qu'ils ont eux mêmes installés là. Les liens de sujétion qu'ils ont entretenus avec les ascendants du lignage qui assure la chefferie du village de Marka-gounko, leur interdisent d'adhérer à cette commune.

Au-delà de cette opposition des Coulibaly de Fougani aux résultats du découpage, l'analyse permet de voir d'autres facettes de la situation conflictuelle créée par le processus de décentralisation. En effet, au niveau village, au sein des communautés elles-mêmes, le conflit existe entre les détenteurs de la chefferie du village, partisans de l'ordre ancien et les groupes sociaux. L'exemple de Tinkolé est assez édifiant dans ce sens. La chefferie y est détenue par les Keita. Les Mariko, Coulibaly, Traoré, Kané,Bagayoko, Diarra et Diallo, sont des résidants qui ont bénéficié de leur hospitalité. Les Keita estiment que tout exercice du pouvoir à la base relève de leurs prérogatives et qu'ils sont par conséquent incontournables dans le choix des futurs responsables de la commune. Les prises de décisions se sont toujours opérées dans leur vestibule, même si par respect ils y ont souvent associé les autres, ils ne sont pas prêts à tolérer une compétition ouverte avec eux. Quant aux hôtes, ils sont de plus en plus conscients de l'opportunité que leur offre la décentralisation dans la conquête du pouvoir. Le code électoral permet à tout un chacun d'être candidat aux compétitions pour assumer des responsabilités dans la municipalité quelle que soit son origine sociale. La 
décentralisation, est une dimension essentielle de tout régime qui se veut véritablement démocratique, car elle consacre la participation des populations à la base, aux prises de décisions donc à l'exercice du pouvoir politique. Si les tenants de la chefferie du village semblent dire oui à la décentralisation, ils sont contrariés par le caractère novateur du mode d'accès au pouvoir. C'est pour cette raison qu'ils s'investissent dans des partis politiques comme l'Adema, le Bdia l'Us-rda, etc, pour barrer route aux autres groupes sociaux. De ce point de vue, les compétitions pour les municipales risquent de donner lieu à des empoignades intéressantes à suivre et à analyser. Un autre aspect de cette situation conflictuelle est l'opposition entre aînés et cadets. Cette opposition vient se superposer aux rapports conflictuels, qu'évoque Claude Meillassoux (1977: 69).

Nous désignons par aînés, tous ceux qui ont accès au vestibule du chef de village pour les prises de décisions, et par cadets, les jeunes et les femmes. Il existe une discrimination assez prononcée vis à vis des jeunes et les femmes. Ceux-ci n'ont pas accès au vestibule ou les décisions sont prises, et c'est là que les premières informations concernant la décentralisation ont été données. Ce groupe social se trouve de fait exclu du pouvoir de décision. Cependant, les deux groupes savent que le contexte politique offre aux jeunes et aux femmes, des possibilités d'accès au pouvoir dans le cadre des municipalités. Le statut social fondé sur l'aînesse est-il suffisant pour assumer des responsabilités dans la commune, pour la gérer convenablement? En tout cas les jeunes, surtout les néo-alphabétisés, pensent pouvoir assumer des responsabilités. Certains auraient déjà fait l'objet de menaces à peine voilées. Cela montre l'importance des enjeux de cette réforme socio-politique.

Les problèmes que nous venons d'évoquer dans la commune de Tinkolé, particulièrement liés à l'ordre ancien, à savoir, aux pratiques socio-politiques anciennes, existent dans beaucoup d'autres au Mali. Il est prévisible qu'ils exerceront une influence préjudiciable au bon fonctionnement des communes rurales.

Conclusion

Le processus de décentralisation vise une réforme qui suscite beaucoup d'espoir chez les populations rurales. Cependant, il apparaît nécessaire de faire preuve de patience et de pédagogie pour leur permettre de mieux comprendre les tenants et les aboutissants de cette entreprise. Ainsi, elles pourront envisager leur avenir avec moins de confusion, tout en mesurant à leur juste valeur certains aspects de leur passé socio-politique.

Il est clair que la décentralisation offre des avantages aux populations, si elle est bien menée. Sur le plan politique, il est réconfortant après avoir passé des années à recevoir les décisions de quelqu'un d'autre et à les appliquer, de pouvoir réfléchir et de choisir ce qui est bon pour soi. S'agissant du domaine socio-économique, les populations à la base sauront désormais comment les ressources qu'elles génèrent sont employées. La décentralisation est certainement une réforme à laquelle il faut adhérer, mais toute précipitation dans ce domaine devrait être évitée car elle risque de bouleverser de manière brutale toute l'organisation sociale au niveau du village, profondément ancrée dans le milieu rural. Une étude initiée par la Mission de décentralisation avec la collaboration de la Coopération allemande (GTZ), les Nations Unies (PNUD) et exécutée par DIRAS SET (Bureau d'études) intitulée: Un découpage territorial au service du développement décentralisé (1994: 105) montre "qu'au-delà de toutes les phases historiques de décomposition et de recomposition du territoire Malien, il y a une constante qui a résisté à tous les changements politiques, administratifs, économiques 
socio-éthniques, c'est l'organisation en village. Cette forme communautaire d'échange, d'appropriation, de solidarité et de hiérarchie sociale est profondément ancrée et continue à régir l'essentiel de la vie socio-économique du Mali". La décentralisation est certainement un cadre favorable au développement des communautés villageoises. Il est nécessaire de prendre le temps, de mieux préparer les populations aux transformations indispensables à opérer. Toute action pouvant déboucher sur un bouleversement mal contenu fragiliserait d'avantage l'organisation sociale villageoise et les structures familiales, déjà éprouvées par l'introduction des cultures de rente dans le système de production dans cette zone, rendrait le problème foncier plus aigu. En effet, la désagrégation des structures familiales ne ferait qu'augmenter le besoin des terres de culture, et mettrait en péril les différentes formes d'associations (tons villageois, associations villageoises) qui ont permis aux communautés villageoises de mettre en place certaines infrastructures, comme les centres de santé, les écoles, les centres d'alphabétisation, les magasins de stockage, etc.

Par contre, les populations seraient prêtes à adopter une réforme qui mettrait à leur disposition un cadre institutionnel garantissant leur pouvoir de décision, concernant les différents aspects de leur développement socio-économique. Une décentralisation bien menée répondrait à un tel souci et affaiblirait l'ordre ancien dans ses aspects rétrogrades.

\section{BIBLIOGRAPHIE}

Brasseur G. 1974. "Le Mali". Notes et Etudes Documentaires 4081-4082-4083 (22 avril 1974).

Journal officiel (n¹) 1997. "Loi n97-008 du 14 janvier 1997 portant loi électorale".

Journal officiel (spécial n 3) 1995. "Textes législatifs et réglementaires", octobre 1995.

Journal officiel (spécial n³) 1996. "Loi n 96-059 du 4 novembre 1996 portant création de communes".

Meillassoux, C. 1977. Terrains et Théories. Anthropos : Paris.

Mission décentralisation 1994. Un découpage territorial au service du développement décentralisé. Bamako : MDD.

Monteil, C. 1977. Les Bambara de Segou et du Kaarta. Paris : Maisonneuve \& Larousse.

Vengroff, R. 1993. La Décentralisation en Afrique : Revue de la littérature.

\section{AUTEUR}

\section{SOLI KONÉ}

Sociologue. Université du Mali, DER Sciences Sociales, ENS, Bamako (Mali). Tél. : 223/22 2189. 